\title{
ME-143 Is Superior to Genistein in Suppression of WNT Signaling in Colon Cancer Cells
}

\author{
SOFYA PINTOVA, KESTUTIS PLANUTIS, MARINA PLANUTIENE and RANDALL F. HOLCOMBE
}

\begin{abstract}
Department of Medicine, Division of Hematology and Medical Oncology, Mount Sinai Medical Center, Tisch Cancer Institute, New York, NY, U.S.A.
\end{abstract}

\begin{abstract}
Background: This study tested the effect of the soy isoflavones genistein and ME-143, and two chemotherapeutic agents, 5-fluorouracil (5FU) and oxaliplatin, on WNT signaling. Materials and Methods: Colon cancer cell lines RKO (hereditary nonpolyposis colorectal cancer type) and DLD1 (most common colorectal cancer type driven by a mutation in WNT pathway) were utilized. WNT throughput was measured using a $\beta$-catenin-responsive SuperTopFlash luciferase assay. A stabilized $\beta$-catenin construct was employed to test $\beta$-catenin involvement in the mechanism of drug activity. Results: ME-143 was a more than 10-fold potent inhibitor of DLD1 proliferation than genistein at $3.125 \mu M$. Genistein alone did not inhibit WNT signaling in either cell line. In RKO cells, oxaliplatin and its combination with $5 F U$ significantly inhibited WNT throughput. Neither $5 F U$, oxaliplatin nor their combination inhibited WNT signaling in DLDI cells. In both the RKO and DLDI cell lines, ME-143 significantly reduced WNT throughput by $65-75 \%$. The introduction of stabilized $\beta$ catenin attenuated the ME-143-dependent inhibition of the $W N T / \beta$-catenin pathway. Conclusion: ME-143 alone and in combination with $5 F U$ and oxaliplatin effectively inhibits the $W N T / \beta$-catenin pathway in colorectal cancer cells of diverse genetic background. $\beta$-Catenin is directly involved in the mechanism of inhibition, and clinical studies are warranted.
\end{abstract}

Genistein, a component of soy, was first isolated from the plant Genista tinctoria in 1899 and chemically synthesized in the 1920s. It is an isoflavone and phytoestrogen found predominantly in soy products that has been studied for its potential role in colon cancer prevention and treatment. In animal models, rats consuming a high genistein diet were

Correspondence to: Sofya Pintova, MD, Mount Sinai Medical Center, Tisch Cancer Institute, One Gustave L Levy Place, Box 1128, New York, NY 10029, U.S.A. Tel: +1 2128248858, Fax: +1 6465379639, e-mail: sofya.pintova@mssm.edu

Key Words: Isoflavone, ME-143, genistein, colon cancer, 5FU, oxaliplatin, cancer cell lines, WNT signaling. reported to develop fewer neoplastic features in their colons and to down-regulate WNT signaling $(1,2)$ leading to the postulation that perhaps genistein and other phytoestrogens may play an antiproliferative role in colon cancer.

Epidemiological studies suggest that a high soy intake, likely owing to its estrogenic properties, may lead to a lower rate of colorectal cancer (3-5). Estrogen supplementation in women has been shown to attenuate the risk of colorectal malignancies (6), suggesting that the antineoplastic process may at least in part be hormone-mediated.

ME-143 is a second-generation synthetic isoflavone that is known to have in vitro anticancer properties and has been shown to inhibit mouse tumor xenograft growth (7). ME-143 was reported to be well-tolerated in a phase I dose-escalation clinical trial in patients with advanced solid tumors (7). Three out of 18 patients in that trial were observed to have stable disease while being treated with single-agent ME-143, which was administered as an intravenous formulation.

It is known that $85 \%$ of colorectal neoplasms (8) have constitutive activation of the WNT pathway. WNT signaling is initiated either extracellularly, through the binding of WNT glycoproteins to a transmembrane frizzled receptor and lowdensity lipoprotein receptor-related protein $5 / 6$ co-receptor (9), or intracellularly, through inactivation of a $\beta$-catenin destruction complex, most commonly through mutation of the adenomatous polyposis coli $(A P C)$ gene. This prevents degradation of $\beta$-catenin, allowing its translocation to the nucleus, which promotes transcription of target genes including cyclin D and cellular myelocytomatosis viral oncogene (c-MYC) (9). Prior studies suggest that genistein inhibits proliferation of colorectal cancer cell lines, possibly through attenuation of WNT signaling by increasing expression of sFRP2, a frizzled receptor antagonist (10-12).

Standard regimen utilized for the treatment of colorectal malignancies is FOLFOX $(13,14)$, based on chemotherapeutic agents 5-fluorouracil (5FU) and oxaliplatin. Few investigations have assessed the effect of 5FU and oxaliplatin on WNT signaling in colorectal cancer cells. In DLD1 cell line-derived chemotherapy-resistant colon cells, 5FU was found to upregulate WNT signaling (15). WNT signaling blockade has 
been shown to reduce resistance to the antiproliferative effects of chemotherapeutic agents such as 5FU and irinotecan (16). Genistein has also been shown to synergistically inhibit tumor proliferation when combined with chemotherapy. When genistein was added to 5FU, enhanced apoptosis was observed in colon cancer cells (17). Genistein appeared to act synergistically with oxaliplatin to inhibit proliferation in pancreatic cancer cell lines and reduce the size of the pancreatic tumors in mice (18). These data led to a theory that the synergistic effect of genistein with chemotherapeutic drugs may be related to its inhibition of WNT signaling.

To our knowledge, the effect of ME-143 on WNT signaling specifically has not been investigated. In this study, we investigated the effects of ME-143, genistein, 5FU and oxaliplatin on WNT throughput in RKO and DLD1 colon cancer cell lines in order to test the hypothesis that a secondgeneration isoflavone, ME-143, alone and in combination with chemotherapy, is more efficacious than genistein in inhibiting WNT signaling.

\section{Materials and Methods}

Cell lines. Cell lines were purchased from the American Type Culture Collection (ATCC), Manassas, VA, USA. RKO (ATCC CRL2577) is a human colon cancer cell line with microsatellite instability and wild-type $A P C$. RKO cells were grown and maintained in Eagle's minimum essential medium (ATCC), with $10 \%$ fetal bovine serum (FBS; Gibco by Thermo Fisher Scientific, Waltham, MA, USA) and penicillin/streptomycin antibiotics (Thermo Fisher Scientific). DLD1 human colon cancer cell line (ATCC CCL221) has an APC mutation; it was maintained in RPM1 growth medium with $10 \%$ FBS and penicillin/streptomycin. Control L mouse cell line (ATCC CRL264) was maintained in Dulbecco's modified Eagle's medium (Thermo Fisher Scientific) with $10 \%$ FBS and penicillin/streptomycin antibiotics. WNT3A-producing cells (ATCC CRL2647) are stably transfected WNT3A-producing L-cells maintained in Dulbecco's modified Eagle's medium with $10 \%$ FBS, penicillin/streptomycin and $400 \mu \mathrm{g} / \mu \mathrm{l}$ of Geneticin (G418 Sulfate; Gibco), antibiotic used to insure stability of WNT3A expression by these cells. WNTconditioned medium was produced without G418 (19). All cell lines were cultured in a humidified incubator with $5 \% \mathrm{CO}_{2}$ at $37^{\circ} \mathrm{C}$.

Experimental agents. Genistein was obtained as a synthetic powder with $>98 \%$ purity from Sigma-Aldrich (St. Louis, MO, USA) and reconstituted as a stock solution in $1 \%$ dimethylsulfoxide (DMSO). It was utilized at concentrations of 0.001 to $300 \mu \mathrm{M}$ for proliferation experiments and at concentrations of $25,50,75$ and $100 \mu \mathrm{M}$ for WNT throughput studies. ME-143 is a second-generation isoflavone generously provided by MEI Pharma (San Diego, CA, USA) and reconstituted as a stock solution in $1 \%$ DMSO. It was utilized at concentrations ranging from 0.39 to $100 \mu \mathrm{M}$ for proliferation experiments and at $3 \mu \mathrm{M}$ for WNT throughput experiments. 5FU (Sigma-Aldrich) was used at concentrations of 10, 20 and $50 \mu \mathrm{M}$, and oxaliplatin (Sigma-Aldrich) was at concentrations of 5 and $10 \mu \mathrm{M}$.

Cell proliferation study. RKO and DLD1 were plated at $10 \%$ confluence in 96-well plates and grown for $24 \mathrm{~h}$, then exposed to either WNT3A-conditioned media to augment WNT signaling or control L-cell-conditioned media for another $24 \mathrm{~h}$. Cell lines were treated with different concentrations of genistein or ME-143. After $72 \mathrm{~h}$, proliferation was measured using MTT assay. Cells were treated with $100 \mu \mathrm{l} /$ well of 3-(4,5-dimethylthiazol-2-yl)-2,5diphenyltetrazolium bromide (MTT) assay reagent and incubated for $2 \mathrm{~h}$ at $37^{\circ} \mathrm{C}$. The plate was then centrifuged at $2,000 \times g$ for $5 \mathrm{~min}$ and the medium was removed. DMSO was then added to solubilize the MTT crystals. Spectophotometric absorbance was measured via a plate reader at wavelength of $570 \mathrm{~nm} \mathrm{(20).}$

WNT throughput assay. Cells were transfected at $\sim 60 \%$ confluence with Renilla luciferase expression construct (10\% of the DNA; Promega Corporation, Madison, WI, USA) and the $\beta$-cateninresponsive firefly luciferase plasmid SuperTOPflash (90\% of DNA; kindly provided by Dr. Randall Moon, University of Washington, Seattle, WA, USA), using transfection methods based on Lipofectamine 2000 (Invitrogen, Carlsbad, CA, USA) for RKO cell line or BioT (Bioland Scientific, Paramount, CA, USA) for DLD1 cells according the manufacturers' instructions. After $48 \mathrm{~h}$, cells were removed from the 6-well plates with EDTA only (no trypsin usage) and plated in 96-well plates in quadruplicates. Both RKO and DLD1 transfected cells were co-cultured with WNT3A-producing L-cells. After $24 \mathrm{~h}$, once the cells had attached, they were treated with genistein, 5FU, oxaliplatin with/without ME-143 with appropriate DMSO control concentrations. After incubating in treatment solutions for $72 \mathrm{~h}$, cells in $50 \mu \mathrm{l} /$ well of media were treated with 50 $\mu 1 /$ well of Dual-Glo Firefly Luciferase substrate (Promega) according to the manufacturer's instructions. WNT throughput activity was then measured by a luminometer (Glomax Multi Detection System; Promega). The Stop \& Glo reagent $(50 \mu \mathrm{l} /$ well) was then added to initiate constitutively expressed Renilla luciferase activity. The ratio of firefly luciferase activity to Renilla luciferase activity was then calculated to normalize the results for transfection efficiency and cell survival. Experiments were subsequently repeated to include transfection of a stabilized murine $\beta$-catenin plasmid with a mutated glycogen synthase kinase 3 beta (GSK3- $\beta$ )-recognition sites, obtained courtesy of Dr. Rolf Kemler, Max Planck Institute of Immunobiology and Epigenetics, Freiburg, Germany.

Statistical analysis. Means and standard error of the mean (SEM) for data from all experimental groups were calculated and groups were compared using a two-tailed unpaired $t$-test with the level of significance defined as $\alpha<0.05$.

\section{Results}

Effect of genistein and ME-143 on proliferation. Genistein inhibited proliferation of RKO cells at concentrations above $10 \mu \mathrm{M}$, with a half maximal inhibitory concentration $\left(\mathrm{IC}_{50}\right)$ of $50 \mu \mathrm{M}$. For DLD1, proliferation was inhibited above $50 \mu \mathrm{M}$, with an $\mathrm{IC}_{50}$ of $75 \mu \mathrm{M}$ (Figure 1). ME-143 was significantly more potent, reducing proliferation of DLD1 cell line by approximately $40 \%$ at a concentration of $3.125 \mu \mathrm{M}$. DLD1 cell line is WNT pathway-driven, like $80 \%$ of colorectal cancer cases.

Effect of genistein and ME-143 on WNT throughput. RKO and DLD1 cells were co-cultured with WNT3A-producing L-cells 


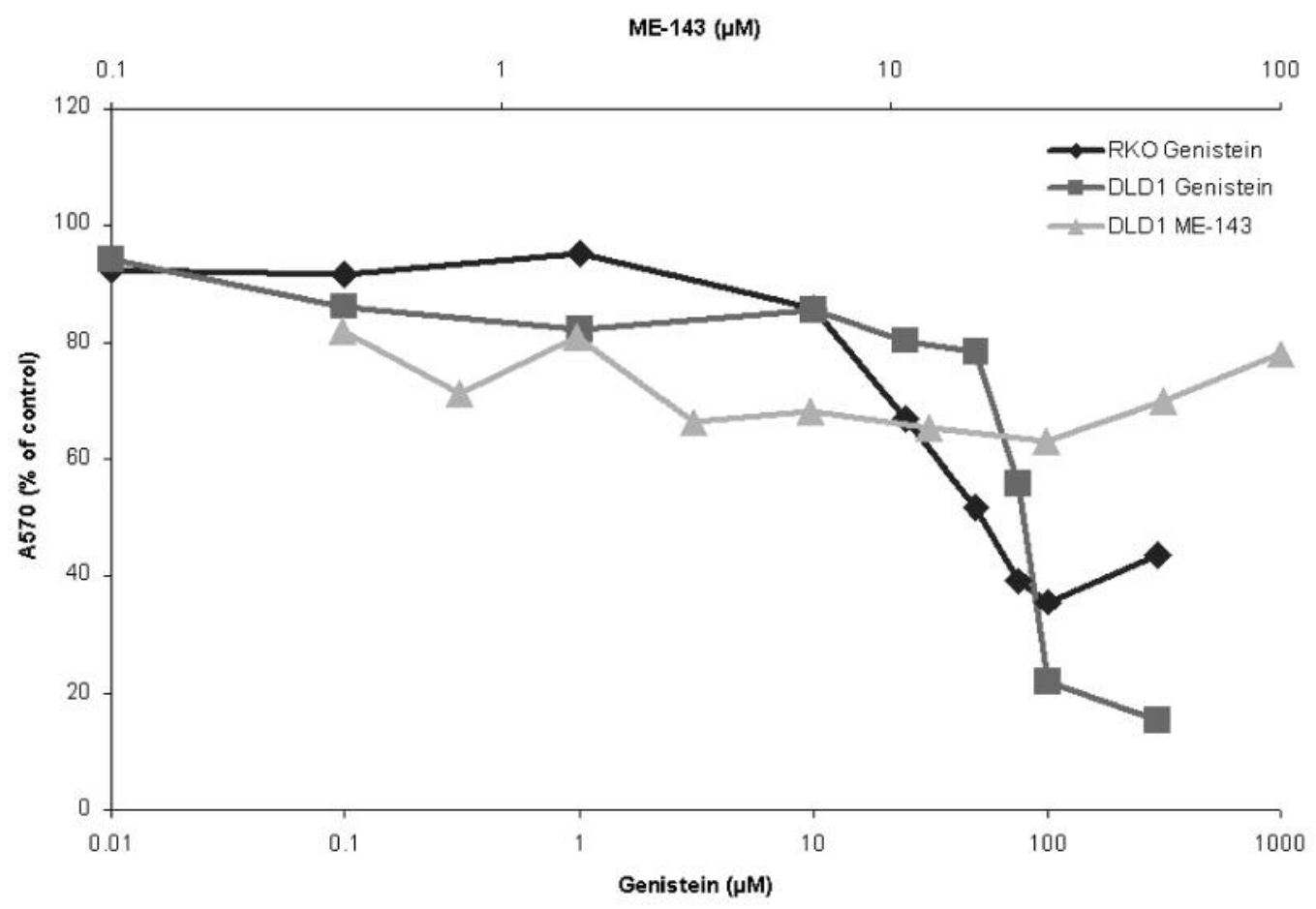

Figure 1. Both genistein and ME-143 inhibited proliferation of colon cancer cells. Proliferation of DLD1 and RKO cells was tested after treatment with concentrations ranging from 0.01 to $300 \mu \mathrm{M}$ of genistein for $72 \mathrm{~h}$ and for DLD1 with ME-143 0-100 $\mu \mathrm{M}$ for $48 \mathrm{~h}$. Genistein had a half-maximal inhibitory concentration $\left(I C_{50}\right)$ of about $75 \mu \mathrm{M}$ for DLD1 cells and approximately $50 \mu M$ for RKO cells. The IC 50 of ME-143 for DLD1 cell line was $3.125 \mu M$. ME-143 was a more than 10-fold potent inhibitor of WNT pathway-driven (as in 80\% of colorectal cancer cases) cell line DLD1 proliferation than genistein. The data for ME-143-treated RKO cells are not available.

to ensure availability of extracellular WNT3A ligands. In both cell lines, genistein had no inhibitory effect on WNT signal throughput at concentrations between $25 \mu \mathrm{M}$ and $100 \mu \mathrm{M}$ (Figure 2). In contrast, ME-143 significantly inhibited WNT/ $\beta$-catenin signaling both in the APC wild-type RKO cell line and in the $A P C$-mutated DLD1 cell line at a concentration of $3 \mu \mathrm{M}(p<0.0001$ and $p=0.0128$, respectively; Figure 3$)$.

Effect of genistein combined with chemotherapeutic agents on WNT throughput. In RKO cells, 5FU and oxaliplatin each reduced WNT throughput slightly in comparison to control but these changes were statistically significant only for oxaliplatin at $5 \mu \mathrm{M}$ (Figure $2 \mathrm{~A} ; p<0.001$ ). However, the combination of $5 \mathrm{FU}$ and oxaliplatin did result in a significant reduction of WNT signaling compared to control (Figure 2A; $p$-values ranging from $<0.05$ to $<0.0001)$ and to oxaliplatin alone ( $p$ values ranging from $<0.05$ to $<0.0001$ ). Genistein significantly augmented inhibition of WNT throughput by $20 \mu \mathrm{M} 5 \mathrm{FU}$ $(p<0.01)$ and of $10 \mu \mathrm{M}$ oxaliplatin $(p<0.0001)$, but did not have a significant effect at other chemotherapy concentrations. Importantly, the addition of genistein to the combination of $5 \mathrm{FU}$ and oxaliplatin did not produce any additional inhibition of WNT signaling (Figure 2A and Figure 4A; $p>0.05$ ).
In DLD1 cells, neither 5FU nor oxaliplatin caused a decrease in WNT throughput as single agents (Figure 2B). WNT throughput was also not suppressed by the combination of 5FU and oxaliplatin in this cell line. Genistein had no inhibitory effect on WNT signaling when combined with 5FU nor with oxaliplatin, nor their combination.

Effect of ME-143 combined with chemotherapeutic agents on WNT throughput. In the RKO cell line, ME-143 alone significantly inhibited WNT signaling and a similar degree of inhibition was seen with the addition of 5FU, oxaliplatin, and their combination (Figure 3A).

However, ME-143 augmented the slight inhibition seen with oxaliplatin alone (Figure 4A; $p<0.001$ ) and the inhibition seen with the $5 \mathrm{FU}$ and oxaliplatin combination (Figure 4A; $p<0.05$ ).

In DLD1 cells, the reduction of WNT signaling by ME-143 appeared to be abrogated by $5 \mathrm{FU}$ as a single agent and in combination with oxaliplatin (Figure 3B; $p$-values range from $<0.01$ to $<0.001$ ). The addition of a higher dose of oxaliplatin alone did not reverse the effect of ME-143, making higher dose oxaliplatin combination with ME-143 most promising for exploring blockade of deleterious effects of high WNT 

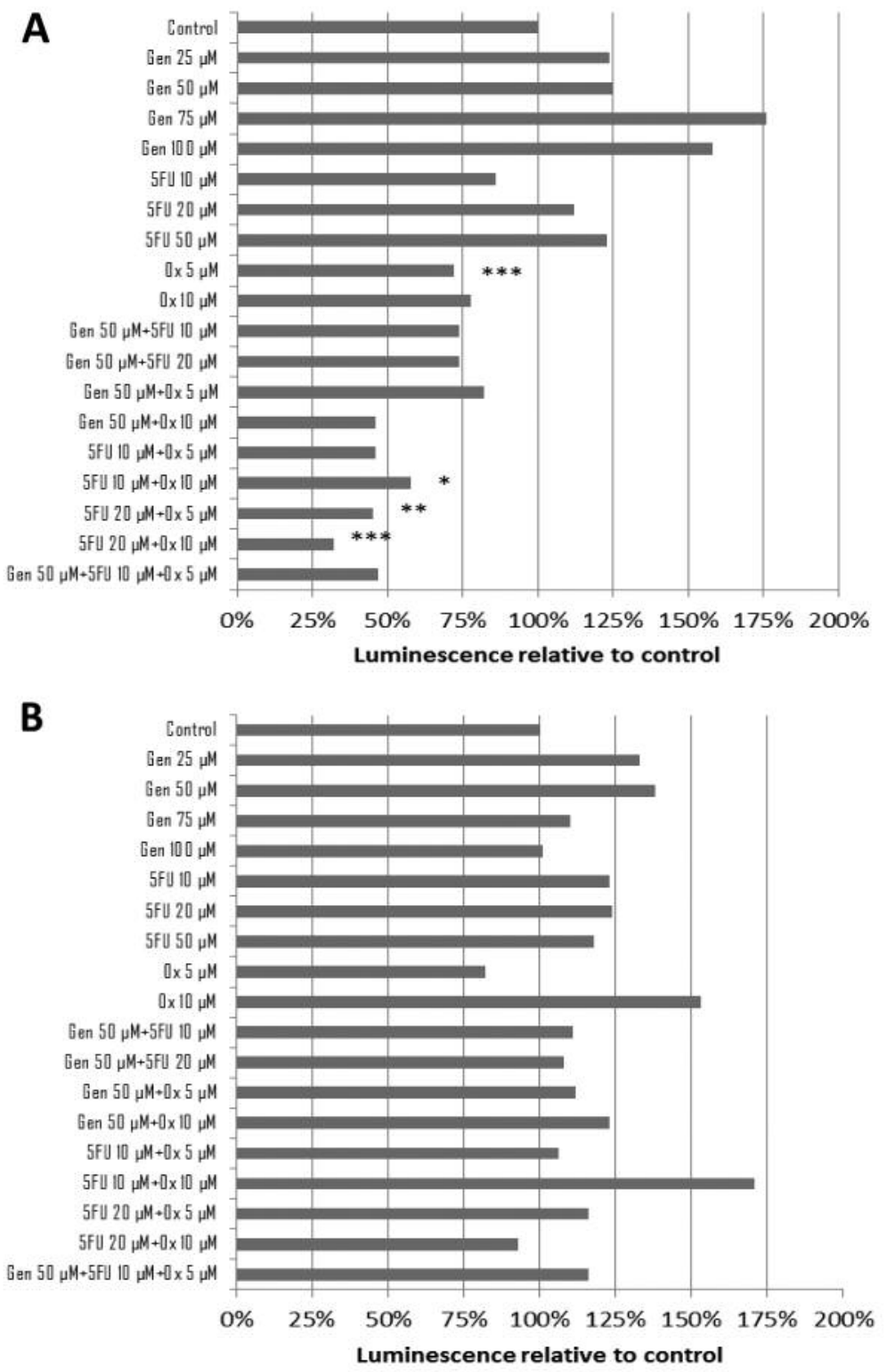

Figure 2. Effect of genistein (Gen), 5-fluorouracil (5FU) and oxaliplatin (Ox) on WNT throughput in RKO and DLD1 colon cancer cells. All wells contained RKO or DLD1 cells co-cultured with WNT3A-producing cells. A: RKO cell line: Genistein failed to produce an inhibitory effect on WNT throughput. Oxaliplatin at $5 \mu \mathrm{M}$ and combinations of $5 F U$ and oxaliplatin with and without genistein produced significant inhibition of WNT throughput. Addition of $50 \mu \mathrm{M}$ genistein to $20 \mu \mathrm{M} 5 \mathrm{FU}$ produced further significant inhibition compared with the same concentration of $5 F U$ alone $(p=0.0094)$. Addition of genistein at $50 \mu \mathrm{M}$ to oxaliplatin at $10 \mu \mathrm{M}$ further enhanced the inhibitory effect on WNT compared to oxaliplatin alone at $10 \mu M(p<0.0001)$. B: DLD1 cell line: Genistein, 5FU and oxaliplatin separately, or combination of $5 F U$ and oxaliplatin all failed to produce significant Wnt throughput inhibition. Significantly different from the control at $* p<0.05$, $* *<<0.001$, and $* * * p<0.0001$.

throughput in most colorectal cancer cases with mutations of WNT pathway components (similar to DLD1 case). When adding ME-143 to 5FU, oxaliplatin and the combination of 5FU and oxaliplatin, considerable inhibitory effects were seen (Figure 4B).

Mechanism of ME-143 inhibition of WNT throughput. Transfection with stabilized $\beta$-catenin was utilized in order to define whether the WNT signaling inhibition by ME-143 occurred upstream or downstream of $\beta$-catenin in the pathway. WNT signaling was markedly reduced in shamtransfected RKO cells upon exposure to ME-143. Transfection with the active construct increased the basal level of WNT throughput in RKO cells. However, the marked inhibition of WNT signaling by ME-143 was almost 

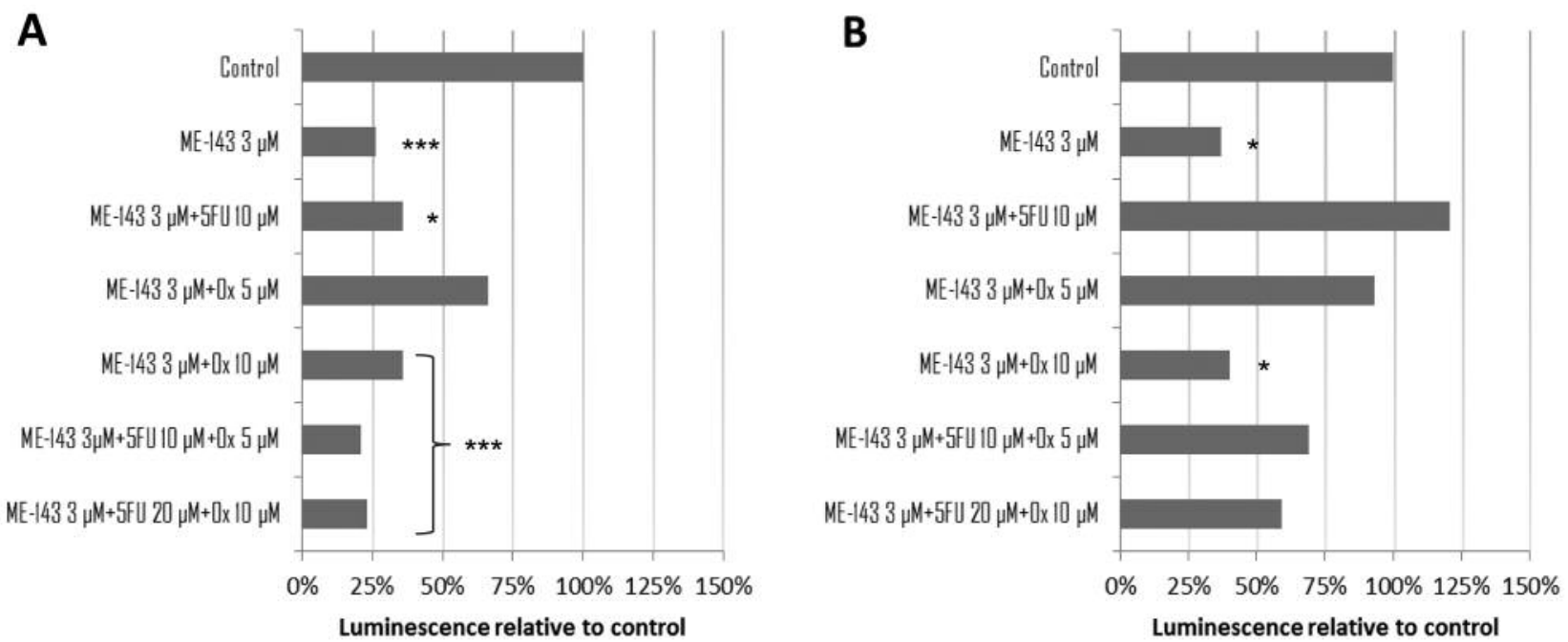

Figure 3. ME-143 generally reduces WNT throughput in RKO and DLD1 colon cancer cells by 65-75\%. All wells contained RKO or DLD1 cocultured with WNT3A-producing cells. A: RKO cell line: ME-143 significantly suppressed WNT throughput alone and in combination with chemotherapy, except when combined with oxaliplatin $(O x)$ at $5 \mu M$. B: DLD1 cell line: ME-143 suppressed WNT throughput but chemotherapy agents restored WNT signaling when combined with ME-143, except for oxaliplatin at $10 \mu M$. Significantly different from the control at $* p<0.05$, $* * p<0.001$, and $* * * p<0.0001$.

A

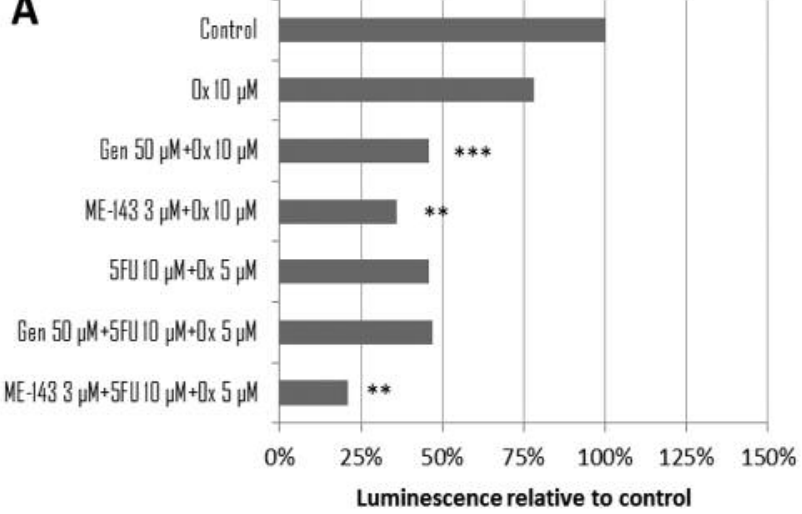

B

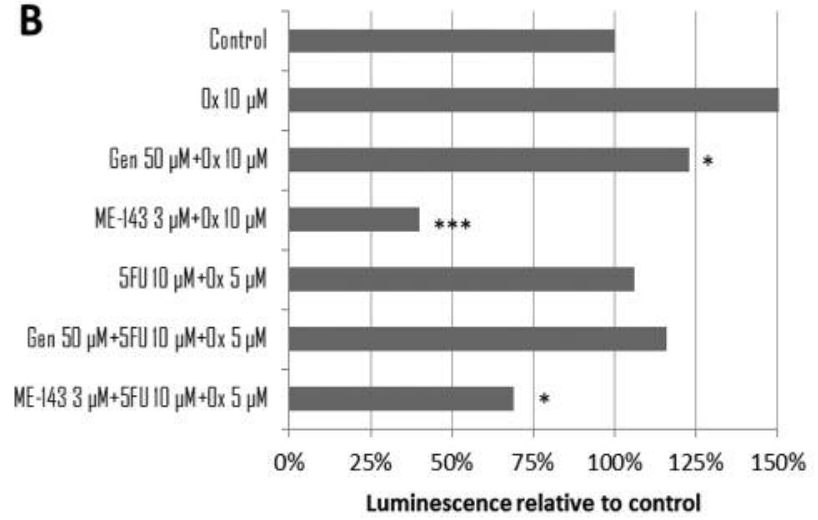

Figure 4. ME-143 provides more potent inhibition of WNT throughput than genistein additional to that of 5-fluorouracil (5FU) and oxaliplatin combination (components of FOLFOX therapy). A: RKO cell line: Addition of $50 \mu M$ genistein (Gen) to $10 \mu M$ oxaliplatin (Ox) further enhanced the inhibitory effect on WNT signaling compared with oxaliplatin alone at $10 \mu M(p<0.0001)$. Genistein failed to provide inhibition of WNT throughput additional to that of $5 F U$ and oxaliplatin combination $(p=0.9191)$. ME-143 further inhibited WNT throughput when added to oxaliplatin at $10 \mu M$ $(p=0.0008)$ and when added to $10 \mu \mathrm{M} 5 \mathrm{FU}$ plus $5 \mu \mathrm{M}$ oxaliplatin $(p=0.0002)$. B: DLD1 cell line: Both genistein and ME-143 produced additional inhibition of WNT throughput compared to oxaliplatin alone at $10 \mu M(p=0.0298$ and $p<0.0001$, respectively). Genistein failed to produce inhibition additional to that of $5 F U$ and oxaliplatin combination ( $p=0.5945)$. ME-143 further inhibited WNT throughput in combination with $10 \mu M$ 5FU plus $5 \mu M$ oxaliplatin compared with the combination alone ( $p=0.0497$ ). Significantly different from the control at $* p<0.05, * * p<0.001$, and $* * * p<0.0001$.

completely abrogated following transfection of stabilized $\beta$ catenin (Figure 5).

\section{Discussion}

In our study, genistein alone failed to produce any inhibition of the WNT pathway in either RKO or DLD1 cell lines.
Genistein has been reported to down-regulate WNT signaling in the colorectal cancer cell line DLD1, by other investigators (11). The difference in these results may be attributed to the difference in methodology used to investigate WNT signaling. Zhang and colleagues showed a decrease in nuclear $\beta$-catenin, while our study measured WNT throughput activity through nuclear $\beta$-catenin- 


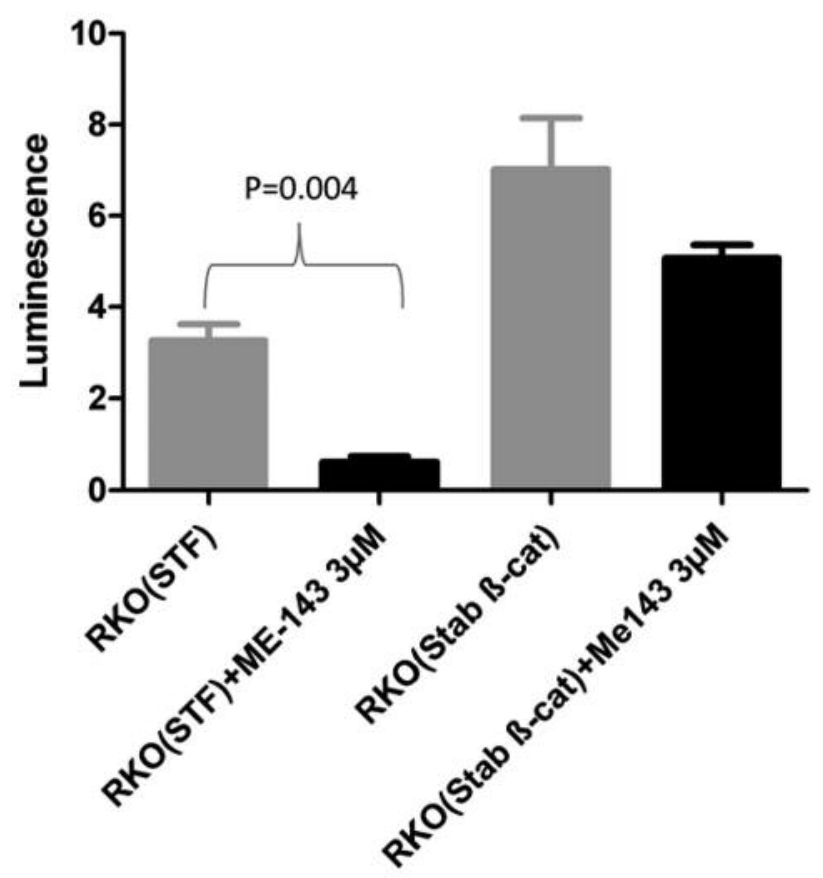

Figure 5. Stabilized $\beta$-catenin attenuates the effect of ME-143 on WNT signaling in the RKO cell line. All wells were co-cultured with WNT3Aproducing cells. Cells were treated for $72 \mathrm{~h}$. RKO cells transfected with reporter plasmid SuperTopFlash (STF) were compared with RKO cells transfected with STF and treated with ME-143; ME-143 significantly inhibited WNT throughput. RKO cells transfected with stabilized $\beta$-catenin and treated with ME-143 showed no inhibition of WNT throughput. RKO cells transfected with stabilized $\beta$-catenin showed greater activation of WNT throughput than RKO cells transfected with STF $(p=0.0189)$.

dependent reporter in the presence of WNT3A. Both studies, however, demonstrated an antiproliferative effect of genistein in the DLD1 colon cancer cell line (Figure 1) and our study demonstrated that genistein also reduced proliferation in the RKO cell line. The exact mechanism by which genistein achieves its inhibitory effect on proliferation is unknown. It is not possible to speculate about WNT-related growth inhibition mechanisms, since in our experiments, no inhibition of WNT pathway by genistein was detected.

As far as we are aware, this is the first study to demonstrate a significant inhibition of WNT signaling by combination treatments of 5FU and oxaliplatin in the RKO cell line, although this effect was not seen in the DLD1 cell line. The concentrations of $5 \mathrm{FU}(10,20$ and $50 \mu \mathrm{M})$ and oxaliplatin $(5$ and $10 \mu \mathrm{M})$ chosen for this study were based on previously published investigations demonstrating their antiproliferative effect in cancer cell lines $(17,18)$. It is possible that to observe an inhibitory effect on WNT signaling in the DLD1 cell line, higher concentrations of 5FU and oxaliplatin combination are required. Genistein did not further augment WNT throughput inhibition when added to the combination of 5FU and oxaliplatin. This is in contrast to prior reports suggesting synergy of genistein with fluoropyrimidine and platin-class chemotherapies (18), although these prior studies were not focused on the effect on WNT signal throughput.

To our knowledge, this is the first study demonstrating significant WNT-throughput inhibition by ME-143, a secondgeneration synthetic isoflavone, in colorectal cancer cell lines. ME-143 was able to achieve this inhibitory effect at 10 -fold lower concentrations than genistein and had a significant effect as a single agent. Of great clinical interest, ME-143 augmented the reduction of WNT signaling observed with the combination of $5 \mathrm{FU}$ and oxaliplatin chemotherapeutic agents (components of the FOLFOX combination). In the DLD1 cell line, $5 \mathrm{FU}$ appeared to be antagonistic to the WNT-inhibitory effect of ME-143. These data suggest that the combination of ME-143 with either 5FU or FOLFOX may be more active for microsatellite-unstable colorectal tumors, where fewer APC mutations are detected (21). Still, it is tempting to speculate that ME-143 could be used clinically for sensitive cells in metastatic disease as a single agent or in combination with FOLFOX. Given its potent effects on WNT signaling, we hypothesize that ME-143 may have its greatest consequence on dormant cancer stem cells which rely on WNT signaling for survival (22). Future studies could investigate the potential role of ME-143 in the adjuvant setting for colorectal cancer as treatment of minimal residual disease. To address residual disease systematically, characteristics of cells responsible for it should be examined. Considering the multigenetic nature of colorectal cancer, preference for a single marker of those cells responsible for the residual disease, such as leucine rich repeat containing G proteincoupled receptor 5 (LGR5), does not look promising (23), and analysis of activity in regulatory pathways including WNT signaling pathway seems to be more desirable.

The attenuation of the inhibition of WNT signaling by ME-143 in cells transfected with stabilized $\beta$-catenin suggests that ME-143-mediated inhibition of WNT pathway indeed acts through the blockade of the $\mathrm{WNT} / \beta$-catenin signaling. We may postulate that the mechanism by which ME-143 inhibits WNT/ $\beta$-catenin pathway is upstream of $\beta$ catenin in the WNT pathway. This may be through increased production of extracellular WNT inhibitory molecules such as SFRPs as has been suggested by others as a possible mechanism for genistein inhibition (12).

\section{Conclusion}

Genistein, at the concentrations used in this study, failed to effectively block the WNT pathway. To our knowledge, this is the first study to demonstrate that ME-143 is a potent inhibitor of the WNT/ $\beta$-catenin pathway in colorectal cancer cell lines at concentrations at least 10 -fold lower than those 
of genistein required to inhibit the growth of cells. The combination of 5FU and oxaliplatin (components of FOLFOX) significantly inhibited WNT signaling in the RKO cell line and this effect was augmented by ME-143. Introduction of stabilized $\beta$-catenin attenuated the ME-143 inhibitory effect suggesting that the mode of action is upstream of $\beta$-catenin in the pathway. Future clinical studies are needed to examine the role of ME-143 in combination with FOLFOX or as a single agent in advanced colorectal cancer, or in the adjuvant setting as treatment of minimal residual disease.

\section{References}

1 Lechner D, Kállay E and Cross HS: Phytoestrogens and colorectal cancer prevention. Vitam Horm 70: 169-198, 2005.

2 Zhang Y, Li Q, Zhou D and Chen H: Genistein, a soya isoflavone, prevents azoxymethane-induced up-regulation of $\mathrm{WNT} / \beta$-catenin signalling and reduces colon pre-neoplasia in rats. Br J Nutr 109(1): 33-42, 2013.

3 Wu AH, Ziegler RG, Horn-Ross PL, Nomura AM, West DW, Kolonel LN, Rosenthal JF, Hoover RN and Pike MC: Tofu and risk of breast cancer in Asian-Americans. Cancer Epidemiol Biomarkers Prev 5(11): 901-906, 1996.

4 Flood DM, Weiss NS, Cook LS, Emerson JC, Schwartz SM and Potter JD: Colorectal cancer incidence in Asian migrants to the United States and their descendants. Cancer Causes Control 11(5): 403-411, 2000.

5 Yu Y, Jing X, Li H, Zhao X and Wang D: Soy isoflavone consumption and colorectal cancer risk: a systematic review and meta-analysis. Sci Rep 6: 25939, 2016.

6 Hebert-Croteau N: A meta-analysis of hormone replacement therapy and colon cancer in women. Cancer Epidemiol Biomarkers Prev 7(8): 653-659, 1998.

7 Pant S, Burris HA 3rd, Moore K, Bendell JC, Kurkjian C, Jones SF, Moreno O, Kuhn JG, McMeekin S and Infante JR: A firstin-human dose-escalation study of ME-143, a second generation NADH oxidase inhibitor, in patients with advanced solid tumors. Inves New Drugs 32(1): 87-93, 2014.

8 Miyaki M, Konishi M, Kikuchi-Yanoshita R, Enomoto M, Igari T, Tanaka K, Muraoka M, Takahashi H, Amada Y, Fukayama M, Maeda Y, Iwama T, Mishima $\mathrm{Y}$, Mori $\mathrm{T}$ and Koike $\mathrm{M}$ : Characteristics of somatic mutation of the adenomatous polyposis coli gene in colorectal tumors. Cancer Res 54(11): 3011-3020, 1994

9 Saif MW and Chu E: Biology of colorectal cancer. Cancer 16(3): 196-201, 2010.

10 Qi W, Weber CR, Wasland K and Savkovic SD: Genistein inhibits proliferation of colon cancer cells by attenuating a negative effect of epidermal growth factor on tumor suppressor FOXO3 activity. BMC Cancer 11: 219, 2011.

11 Kim EJ, Shin HK and Park JH: Genistein inhibits insulin-like growth factor-I receptor signaling in HT-29 human colon cancer cells: a possible mechanism of the growth inhibitory effect of Genistein. J Med Food 8(4): 431-438, 2005.

12 Zhang Y and Chen H: Genistein attenuates WNT signaling by up-regulating sFRP2 in a human colon cancer cell line. Exp Biol Med 236(6): 714-722, 2011.
13 Saltz LB, Clarke S, Diaz-Rubio E, Scheithauer W, Figer A, Wong R, Koski S, Lichinitser M, Yang TS, Rivera F, Couture F, Sirzen $\mathrm{F}$ and Cassidy $\mathrm{J}$ : Bevacizumab in combination with oxaliplatin-based chemotherapy as first-line therapy in metastatic colorectal cancer: a randomized phase III study. J Clin Oncol 26(12): 2013-2019, 2008.

14 Andre T, Boni C, Navarro M, Tabernero J, Hickish T, Topham C, Bonetti A, Clingan P, Bridgewater J, Rivera F and de Gramont A: Improved overall survival with oxaliplatin, fluorouracil, and leucovorin as adjuvant treatment in stage II or III colon cancer in the MOSAIC trial. J Clin Oncol 27(19): 3109-3116, 2009.

15 Deng YH, Pu XX, Huang MJ, Xiao J, Zhou JM, Lin TY and Lin E H: 5-Fluorouracil upregulates the activity of Wnt signaling pathway in CD133-positive colon cancer stem-like cells. Chin J Cancer 29(9): 810-815, 2010.

16 Chikazawa N, Tanaka H, Tasaka T, Nakamura M, Tanaka M, Onishi $\mathrm{H}$ and Katano M: Inhibition of Wnt signaling pathway decreases chemotherapy-resistant side-population colon cancer cells. Anticancer Res 30(6): 2041-2048, 2010.

17 Hwang JT, Ha J and Park OJ: Combination of 5-fluorouracil and genistein induces apoptosis synergistically in chemo-resistant cancer cells through the modulation of AMPK and COX-2 signaling pathways. Biochem Biophys Res Commun 332(2): 433-440, 2005.

18 Banerjee S, Kong D, Azmi AS, Wang Z, Ahmad A, Sethi S and Sarkar FH: Restoring sensitivity to oxaliplatin by a novel approach in gemcitabine-resistant pancreatic cancer cells in vitro and in vivo. Int J Cancer 128(5): 1240-1250, 2011.

19 Willert K, Brown JD, Danenberg E, Duncan AW, Weissman IL, Reya T, Yates JR 3rd and Nusse R: Wnt proteins are lipidmodified and can act as stem cell growth factors. Nature 423(6938): 448-452, 2003.

20 Jung DB, Lee HJ, Jeong SJ, Lee EO, Kim YC, Ahn KS, Chen $\mathrm{CY}$ and Kim SH: Rhapontigenin inhibited hypoxia inducible factor 1 alpha accumulation and angiogenesis in hypoxic PC-3 prostate cancer cells. Biol Pharm Bull 34(6): 850-855, 2011.

21 Christie M, Jorissen RN, Mouradov D, Sakthianandeswaren A, Li S, Day F, Tsui C, Lipton L, Desai J, Jones IT, McLaughlin S, Ward RL, Hawkins NJ, Ruszkiewicz AR, Moore J, Burgess AW, Busam D, Zhao Q, Strausberg RL, Simpson AJ, Tomlinson IP, Gibbs $\mathrm{P}$ and Sieber OM: Different APC genotypes in proximal and distal sporadic colorectal cancers suggest distinct $\mathrm{WNT} /$ beta-catenin signalling thresholds for tumourigenesis. Oncogene 32(39): 4675-4682, 2013.

22 Salem ML, El-Badawy AS and Li Z: Immunobiology and signaling pathways of cancer stem cells: implication for cancer therapy. Cytotechnology 67(5): 749-759, 2015.

23 Planutis AK, Holcombe RF, Planoutene M and Planoutis K: SW480 colorectal cancer cells that naturally express Lgr5 are more sensitive to the most common chemotherapeutic agents than Lgr5-negative SW480 cells. Anticancer Drugs 26(9): 942$947,2015$.

Received December 9, 2016

Revised February 26, 2017

Accepted March 2, 2017 